\title{
Checklist for Determining Severity of Pain and Type and Dosage of Analgesics Administered to Patients Undergoing Breast Surgeries
}

\author{
Davoud Aghamohamadi ${ }^{1}$, Mehdi Khanbabayi Gol ${ }^{*}{ }^{\circledR}$
}

\begin{abstract}
Objectives: Controlling acute pains requires proper diagnosis and prescription of appropriate drugs. Hence, it is quite necessary to develop a systematic checklist containing all factors affecting the acute postoperative pain or any acute pain associated with breast cancer in order to determine the type and dosage of analgesics administered to the patients. The aim of the present study was the design, and psychometric and pilot execution of a checklist for determining the severity of pain, as well as the type and dosage of analgesics administered to patients who underwent breast surgeries.

Materials and Methods: The present methodological study was conducted in Tabriz from 2018 to 2019. The participants consisted of 340 patients who were selected based on the inclusion and exclusion criteria. The study was carried out in four stages including interviews with patients and the elicitation of experts' views and opinions, checklist validity assessment, the finalization of the items, and finally, questionnaire reliability assessment. Eventually, the data were statistically analyzed using the inter-cluster correlation test and the Kuder-Richardson Formula 20 (KR-20) in SPSS-19.

Results: In the first stage, a 100-item checklist was designed, which included 4 subscales (i.e., severe, relatively severe pain, moderate, and mild pain). Then, 66 items were eliminated due to the low content validity ratio and content validity index (CVI). The final checklist contained 34 items with an S-CVI/Ave of 0.941 , as well as an inter-cluster correlation test $(\mathrm{P}=0.001)$ and internal consistency of 0.921 .

Conclusions: The final checklist entitled "Pain Diagnosis and Drug Prescription for Breast Cancer Patients" was an appropriate tool for the precise determination of the severity of pain in addition to the type and dosage of postoperative analgesics.

Keywords: Validity, Pain, Checklist, Reliability, Pain control
\end{abstract}

\section{Introduction}

Breast surgeries are associated with severe pain in people with breast cancer. After each surgery such as tumor removal, lymphadenectomy, and partial or complete mastectomy, such patients feel severe pain at the surgery site resulting from surgical incisions and stitching. During chemotherapy and radiotherapy, patients further feel severe edema and swelling in their breast, the axillary lymph nodes, along with the shoulder and arm of the affected hand since they may need invasive treatments or strong painkillers. Given that the survival rate of women with breast cancer is increasing due to the progress in diagnostic and therapeutic approaches, it is quite essential to adopt measures to control postoperative pain $(1,2)$.

Although many drugs and procedures are available for managing postoperative pain, its control is not satisfactory (3). Thus, failure to identify pain intensity by the treatment team reduces the possibility of pain control and causes the patient to experience severe pains. In a study about pain evaluation and control, it was shown that 35\%-55\% of caregivers underestimate the severity of pain (4). Based on the above-mentioned explanation, it is quite necessary to develop a systematic checklist containing all factors affecting acute postoperative pain or any acute pain related to breast cancer in order to identify the type and dosage of analgesics administered to the patients. In addition, the existing checklists are unsuitable for the simplicity of the details and the type of pain that patients are afflicted with and the type of drug they need to receive. The main objective of the present study included the design, and psychometric and pilot execution of a checklist for determining the severity of pain and the type and dosage of analgesics administered to patients undergoing breast surgeries.

\section{Materials and Methods}

The present methodological study (instrumentation and validation) was performed in Tabriz from September 23, 2018, to April 19, 2019. The study population consisted of all women with breast cancer undergoing breast surgeries (i.e., lumpectomy, mastectomy, and lymphadenectomy). The inclusion criteria were suffering from breast cancer 
confirmed by an oncologist and a pathologist, being at least 18 years old, and having a high school diploma or higher degree. On the other hand, the exclusion criterion included unwillingness to participate in the study and the metastasis of other cancers to the breast. The participants were selected using a purposive sampling method, in which samples are selected based on prior data about population characteristics and a defined objective $(5,6)$. Accordingly, the samples are selected from a place or group which is most representative of the population. In the present study, the participants were selected from among the patients visiting Imam Reza Hospital of Tabriz because this hospital accepts many patients as a treatment center. Further, many patients with breast cancer in the northwest of Iran are referred to this hospital and are hospitalized surgically. Considering the study population size and the research objectives, the sample size was determined to be 340 including lumpectomy $(\mathrm{n}=71)$, mastectomy $(\mathrm{n}=150)$, and lymphadenectomy $(\mathrm{n}=119)$ because it should be more than 300 for factor analysis (7).

The studied checklist was tested on the participants as a structured interview about pain and the influential factors. After getting permission from the participants, the interviews were recorded based on the participants' permission for voice-recording. The participants answered the questions during an interview. To ensure the accuracy of the research data, the participants were allowed to have a short rest whenever they felt tired or incapacitated of giving any response.

\section{First Stage}

In this stage, the interviews were analyzed by the research team and the results were recorded in the order of priority. Then, according to the views of pain specialist and palliative care specialists, recent studies on pain-related factors were carefully studied so that they became aware of the latest related articles (10 specialists who were not aware of the interviews and their results), followed by listing the factors affecting pain in patients with breast cancer in a checklist.

\section{Second Stage}

In this stage, the validity of the studied checklist was measured using face, content, and construct validity. To assess face validity, the authors tried to use the correct method of writing and logical wording in order to develop the items. The research team reread the items several times and applied the necessary corrections and then two methodologists reviewed and revised them. The content validity of the checklist was determined by using a quantitative method based on the views of a researcher who had a specialty in tool design, pain, and anesthesiology. Furthermore, this type of validity was measured using content validity ratio (CVR), content validity index (CVI), and scale-level content validity index/averaging calculation method (S-CVI/Ave). To determine CVR, 10 experts were asked to categorize each stage as "necessary", "beneficial", or "unnecessary".

Based on critical values for Lawshe's CVR, items with a CVR value of more than 0.62 (according to the evaluation of 10 experts) were considered significant $(P<0.05)$ and protected as well (8). Then, CVI was determined by using Waltz and Bausell's CVI (9). For this purpose, 15 experts (different from those in the previous stage) were asked to determine the relevancy, simplicity, and clarity of each item in the checklist based on a four-point Likerttype scale (e.g., 1: Not relevant, 2: Relatively relevant, 3: Relevant, and 4: Totally relevant) for the first question. Moreover, CVI value in this study was calculated by dividing the number of experts scoring an item 3 or 4 by the total number of the experts (9). Hyrkäs et al suggested a score of 0.79 or more for the acceptance of the items based on the CVI value (10). Then, the mean S-CVI/Ave of the checklist was calculated based on the mean CVI score of all items. According to Polit and Beck, a score of 0.90 or more means that the S-CVI/Ave is acceptable.

\section{Third Stage}

In the third stage, also called "item reduction" stage, three methodologists specializing in the questionnaire design (other than the research team) were asked to select the items that better convey the concepts and, if necessary, merge two or more items that are related to each other to present a clear and accurate concept. In this stage, the methodologists had relatively similar views about eliminating or merging the items.

\section{Fourth Stage}

In this stage, the reliability of the checklist was assessed using internal consistency and inter-rater reliability. Internal consistency was measured using the KuderRichardson Formula 20 (KR-20), which is a scale of internal consistency reliability for measurements with dichotomous choices. The reliability is acceptable if this coefficient is greater than 0.70 (11). In this study, the interobserver agreement was also calculated to achieve interrater validity. Accordingly, the higher agreement between the scores given by the raters demonstrated higher validity between them. First, the raters were briefed on the checklist application and then each rater was observed and evaluated by two other raters. The agreement between the observers was calculated by using the intraclass correlation coefficient (ICC). It is noteworthy that ICC is the most accepted statistical test for calculating the index reliability. The validity is considered desirable if this index is greater than 0.80 (4).

The number of samples needed to determine the construct validity is different from the viewpoint of the researchers. However, it is recommended that 5-10 samples are appropriate for each item (12). Accordingly, a sample of 10 times of the checklist items was selected in this study. Moreover, random sampling from Imam Reza 
hospital of Tabriz was used for determining the construct validity and reliability of the checklist.

The data were statistically analyzed using the intercluster correlation test and the KR-20 in SPSS-19.

The finalized checklist containing 30 items, was tested in a pilot study. The author completed the checklist for each patient after the surgery when they were fully conscious and able to answer the questions. Then, the required drug was administered to them based on the checklist score, followed by measuring the severity of pain. In addition to the checklist, the visual analogue scale was used to compare the severity of pain before and after taking the drug.

The compliance with ethical considerations was similar to that of the other studies (13-15). The necessary permission for the study was obtained from the authorities of Imam Reza Hospital. The patients were briefed on the research objectives and procedure, and the interviews were recorded after getting permission from the participants. They were also assured that their personal information will be kept confidential and merely used for research purposes. Moreover, the patients were free to withdraw from the study at any stage upon their desire.

\section{Results}

Using the phenomenological research methods in the first stage of the research, the severity of pain and its influential factors were defined based on the experiences of women with breast cancer undergoing breast surgeries. Thus, 4 subscales of pain severity (i.e., severe, relatively severe, moderate, and mild pain) and the factors affecting each subscale were defined in this regard. Then, the checklist items were extracted from thematic phrases obtained from the structured interview of the previous stage. Next, the research team reviewed the extracted items and merged them with overlapping concepts in order to develop an initial 100-item checklist. These 100 items consisted of $31,30,20$, and 19 questions about the causes of severe, relatively severe, moderate, and mild pains, respectively.
In the next stage, 40 and 26 items were excluded from the checklist due to a CVR of less than 0.62 and a CVI of less than 0.79 , respectively. Therefore, according to experts' views, the number of items was reduced to 34 in 4 subscales $(10,7,8$, and 9 items for severe, relatively severe, moderate, and mild pain, respectively), the details of which are provided in Table 1. Each item could be answered as "Yes" or "No", and patients were allowed to add more explanation at the end of each item. "Yes" answers were given a score of 4, 3, 2, and 1 for severe, relatively severe, moderate, and mild pains, respectively, and "No" answers received a zero score. It should be also noted that the S-CVI/Ave of the final checklist items was equal to 0.941 .

In the next stage, the reliability of the checklist was evaluated by using internal consistency and inter-rater reliability. To determine internal consistency, the KR-20 was calculated for the observational checklist on a sample of 340 patients. The results of this study indicated that the internal consistency of the entire scale was 0.921 (Table 2).

Additionally, the results of the inter-cluster correlation test showed that there was a significant agreement between the scores of the first and the second observers $(P<0.001)$. This indicates the agreement between the raters on the subscales and the entire checklist and the optimum reliability of the checklist (Table 3 ).

The obtainable minimum and maximum scores on this scale are equal to 0 and 86 , respectively. The pain can be controlled by taking sedatives, anxiolytics, nonsteroidal anti-inflammatory drugs, anticonvulsants, antidepressants, opioids once a day, opioids twice a day, and opioids three times a day if the score is within the range of $0-12,13-25,26-38,39-51,52-61,62-72,73-80$, or greater than 80 , respectively. The results of this section regarding the type of the drug and its dosage were also based on the severity of pain that was validated in the pilot study. However, further studies are needed in this regard in the future.

Based on the visual analogue scale checklist, the mean

Table 1. Content Validity of the Final Checklist Items

\begin{tabular}{|c|c|c|c|c|c|c|c|c|}
\hline Question Number & CVR & CVI & Question Number & CVR & CVI & Question Number & CVR & CVI \\
\hline Q1 & 0.842 & 0.860 & Q13 & 1 & 0.990 & Q24 & 0.909 & 0.900 \\
\hline Q2 & 0.898 & 0.902 & Q14 & 0.822 & 0.852 & Q25 & 0.957 & 0.961 \\
\hline Q3 & 0.924 & 0.950 & Q15 & 0.899 & 0.905 & Q26 & 0.937 & 0.941 \\
\hline Q4 & 0.991 & 0.990 & Q16 & 0.907 & 0.902 & Q27 & 0.925 & 0.930 \\
\hline Q5 & 0.961 & 0.957 & Q17 & 0.900 & 0.909 & Q28 & 0.951 & 0.950 \\
\hline Q6 & 0.900 & 0.906 & Q18 & 0.922 & 0.915 & Q29 & 0.981 & 0.975 \\
\hline Q7 & 0.995 & 1 & Q19 & 0.889 & 0.909 & Q30 & 0.903 & 0.905 \\
\hline Q8 & 0.985 & 0.927 & Q20 & 0.903 & 0.900 & Q31 & 0.907 & 1 \\
\hline Q9 & 0.900 & 0.901 & Q21 & 0.906 & 0.901 & Q32 & 0.921 & 0.930 \\
\hline Q10 & 0.886 & 0.900 & Q22 & 0.895 & 0.905 & Q33 & 0.907 & 0.905 \\
\hline Q11 & 0.986 & 0.978 & Q23 & 0.978 & 0.977 & Q34 & 0.901 & 0.906 \\
\hline Q12 & 0.906 & 0907 & & & & & & \\
\hline
\end{tabular}

Note. CVR: Content validity rate; CVI: Content validity index. 
Table 2. Internal Consistency of Checklist Factors

\begin{tabular}{lcc}
\hline Sub-scale & $\begin{array}{c}\text { Number and Number of } \\
\text { Phrases }\end{array}$ & KR-20 (N=340) \\
\hline Severe pain & $10-(1-10)$ & 0.904 \\
Relatively severe pain & $7-(11-17)$ & 0.912 \\
Moderate pain & $8-(18-25)$ & 0.919 \\
Mild pain & $9-(26-34)$ & 0.930 \\
Total & $34-(1-34)$ & 0.921 \\
\hline
\end{tabular}

Note. KR: Kuder-Richardson formula.

Table 3. Results of the Inter-cluster Correlation Test

\begin{tabular}{lcccc}
\hline \multirow{2}{*}{ Sub-scale } & \multirow{2}{*}{ ICC } & \multicolumn{2}{c}{$95 \% \mathrm{Cl}$} & \multirow{P}{*}{$\begin{array}{c}\text { Value } \\
\text { (n=30) }\end{array}$} \\
\cline { 3 - 4 } & & Min & Max & \\
\hline Severe pain & 0.977 & 0.950 & 0.999 & 0.001 \\
Relatively severe pain & 0.949 & 0.9925 & 0.963 & 0.001 \\
Moderate pain & 0.968 & 0.949 & 0.979 & 0.001 \\
Mild pain & 0.980 & 0.978 & 0.987 & 0.001 \\
Total & 0.960 & 0.955 & 0.966 & 0.001 \\
\hline
\end{tabular}

Note. ICC: Intraclass correlation coefficient; $\mathrm{Cl}$ : confidence interval; Min: Minimum; Max: Maximum.

severity of postoperative pain was equal to $7.73 \pm 1.62$. After diagnosing the severity of pain based on the designed checklist and prescribing the required drug for each patient, the mean severity of postoperative pain was obtained $2.06 \pm 0.50$. The designed checklist could correctly evaluate the severity of pain in all patients in the pilot study.

\section{Discussion}

A 100-item checklist was developed for the evaluation of postoperative pain severity in patients with breast cancer based on the concept explained in qualitative research. Then, face validity, content validity, internal consistency (KR-20), and inter-observer agreement of the checklist were confirmed based on the findings. It should be noted that the designed checklist is relatively easy to use and all people providing health services in hospitals, medical and rehabilitation centers, and even at home can complete it in 15 minutes. The face and content validity of this checklist indicate the simplicity and clarity of its items.

In this study, CVR and CVI were used to assess the content validity of the checklist and 40 and 26 items were eliminated accordingly. In addition, the S-CVI/Ave was obtained 0.941, which was favorable. According to Polit and Beck, a score of 0.90 or more implies an acceptable S-CVI/Ave (9). Therefore, the designed checklist was in good status in terms of the content. The KR-20 of this checklist suggests appropriate internal consistency of its items and reliability of the entire checklist. Many researchers and incrementors have used and confirmed this tool (16-18).

Since there was no checklist for measuring the severity of pain based on its affecting factors and prescribing the right drug for pain control, the present study aimed to develop such a checklist and test it in a pilot study. The results of pilot execution indicated that this checklist can help us to accurately determine postoperative pain in patients suffering from breast cancer and then prescribe the right drug for pain control, as one of the principles of postoperative care. Moreover, this checklist informs us that there is no need for prescribing opioids that have many side effects when the patient is suffering from a moderate level of pain. Additionally, when the severity of pain is not well diagnosed by medical staff, this checklist helps them to measure the severity of pain and determine the right drug for pain control. Considering that, to the best of our knowledge, this is the first checklist for measuring the severity of pain, it is recommended to test this checklist in several studies to identify and eliminate its weaknesses. The advantage of this checklist over the other checklists is that in previous checklists, there is no need for pain based on the severity of the pain. Therefore, the physicians cannot accurately prescribe the drug to the patient after surgery and complications. This checklist can be used by all members of the treatment team because of its simple questions.

\section{Limitations}

The design of a checklist that emphasizes postoperative pain and identifies the type of pain relief is necessary. All participated patients in this study were from the same region of Iran, which is one of the limitations of this study because people of different cultures and ethnicities have different definitions of the pain. A low level of attendance and the sampling method were the other major limitations of the present study. Therefore, it is recommended to test this checklist on patients of other regions and countries in order to ensure its effective application.

\section{Suggestions for Future Studies}

The other researchers are recommended to use this checklist to evaluate and reinforce its applicability and to identify its potential weaknesses.

Strengths and Weaknesses of the Study

The strengths of this study included the size of the sample and the creation of a tool that can measure the amount of pain and the required medication while the low level of education was considered as the weak point of the study.

\section{Conclusions}

This checklist is unique in its kind as the one which identifies the determinants of postoperative pain and the need for an analgesic drug. The present study managed to develop a checklist for measuring the severity of postoperative pain in patients with breast cancer and to determine the type and dosage of analgesics.

\section{Conflict of Interests}

None. 


\section{Ethical Issues}

The research project was approved by the Ethics Committee of Tabriz University of Medical Sciences (the ethics No. of IR.TBZMED.REC.1397.1059).

\section{Financial Support}

This study was granted by Tabriz University of Medical Sciences.

\section{Acknowledgments}

The authors would like to thank Clinical Research Development Unit, Shohada Hospital, Tabriz University Of Medical Sciences for kind supports. The researchers would like to give their gratitude to the Research Center and the Health Vice-chancellor of Tabriz University of Medical Sciences for financial support.

\section{References}

1. Galvão DA, Newton RU. Review of exercise intervention studies in cancer patients. J Clin Oncol. 2005;23(4):899909. doi:10.1200/jco.2005.06.085

2. Mols F, Vingerhoets AJ, Coebergh JW, van de Poll-Franse LV. Quality of life among long-term breast cancer survivors: a systematic review. Eur J Cancer. 2005;41(17):2613-2619. doi:10.1016/j.ejca.2005.05.017

3. Yalcin N, Uzun ST, Reisli R, Borazan H, Otelcioglu S. A comparison of ketamine and paracetamol for preventing remifentanil induced hyperalgesia in patients undergoing total abdominal hysterectomy. Int J Med Sci. 2012;9(5):327333. doi:10.7150/ijms.4222

4. Aslan FE, Badir A, Selimen D. How do intensive care nurses assess patients' pain? Nurs Crit Care. 2003;8(2):62-67. doi:10.1046/j.1478-5153.2003.00006.x

5. Acharya AS, Prakash A, Saxena P, Nigam A. Sampling: why and how of it. Indian J Med Spec. 2013;4(2):330-333. doi:10.7713/ijms.2013.0032

6. Patton MQ. Sampling, qualitative (purposeful). The Blackwell Encyclopedia of Sociology. 2007.

7. MacCallum RC, Widaman KF, Zhang S, Hong S. Sample size in factor analysis. Psychol Methods. 1999;4(1):84-99.
doi:10.1037/1082-989X.4.1.84

8. Lawshe $\mathrm{CH}$. A quantitative approach to content validity 1. Pers Psychol. 1975;28(4):563-575. doi: 10.1111/j.17446570.1975.tb01393.x

9. Polit DF, Beck CT, Owen SV. Is the CVI an acceptable indicator of content validity? appraisal and recommendations. Res Nurs Health. 2007;30(4):459-467. doi:10.1002/nur.20199

10. Hyrkäs K, Appelqvist-Schmidlechner K, Oksa L. Validating an instrument for clinical supervision using an expert panel. Int J Nurs Stud. 2003;40(6):619-625. doi:10.1016/ s0020-7489(03)00036-1

11. Salkind NJ. Encyclopedia of Research Design. Thousand Oaks: Sage; 2010.

12. de Boer MR, Moll AC, de Vet HC, Terwee CB, VölkerDieben HJ, van Rens GH. Psychometric properties of vision-related quality of life questionnaires: a systematic review. Ophthalmic Physiol Opt. 2004;24(4):257-273. doi:10.1111/j.1475-1313.2004.00187.x

13. Naderpour M, Shahidi N, Hemmatjoo T. Comparison of tympanoplasty results in dry and wet ears. Iran J Otorhinolaryngol. 2016;28(86):209.-214

14. Mobaraki-Asl N, Ghavami Z, Gol MK. Development and validation of a cultural competence questionnaire for health promotion of Iranian midwives. J Educ Health Promot. 2019;8:179. doi: 10.4103/jehp.jehp_185_19.

15. Aghamohammadi D, Gargari RM, Fakhari S, Bilehjani E, Poorsadegh S. Classic versus inguinal approach for obturator nerve block in transurethral resection of bladder cancer under spinal anesthesia: a randomized controlled trial. Iran J Med Sci. 2018 Jan;43(1):75-80.

16. Reis $M$, Nahmiash D. Validation of the indicators of abuse (IOA) screen. Gerontologist. 1998;38(4):471-480. doi:10.1093/geront/38.4.471

17. Cohen M. Research assessment of elder neglect and its risk factors in a hospital setting. Intern Med J. 2008;38(9):704707. doi:10.1111/j.1445-5994.2008.01630.x

18. Cohen M, Halevi-Levin S, Gagin R, Friedman G. Development of a screening tool for identifying elderly people at risk of abuse by their caregivers. J Aging Health. 2006;18(5):660-685. doi:10.1177/0898264306293257

(c) 2020 The Author(s); This is an open-access article distributed under the terms of the Creative Commons Attribution License (http:// creativecommons.org/licenses/by/4.0), which permits unrestricted use, distribution, and reproduction in any medium, provided the original work is properly cited. 